NASA Technical Memorandum 105360

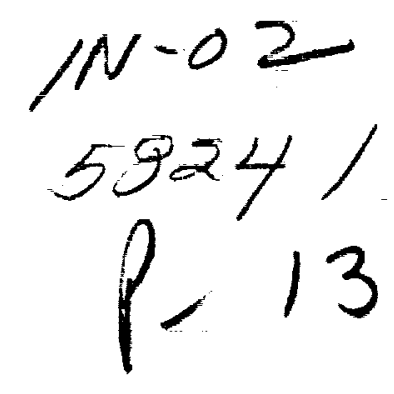

AIAA - 92-0065

\title{
On the Mechanism of Turbulence Suppression in Free Shear Flows Under Acoustic Excitation
}

K.B.M.Q. Zaman and E.J. Rice

Lewis Research Center

Cleveland, Ohio

Prepared for the

30th Aerospace Sciences Meeting and Exhibit

sponsored by the American Institute of Aeronautics and Astronautics

Reno, Nevada, January 6-9, 1992

\section{N/Sก}




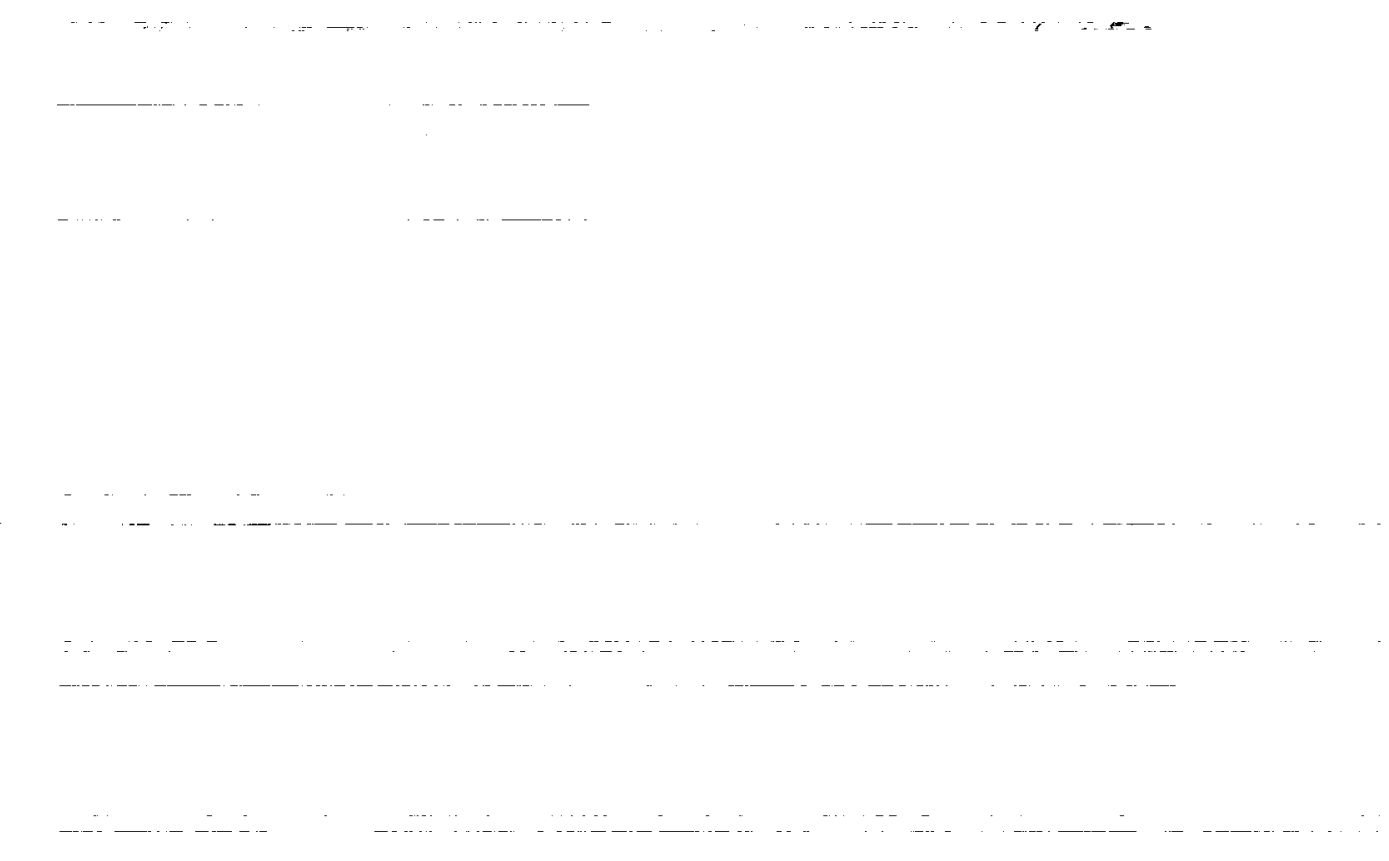




\title{
ON THE MECHANISM OF TURBULENCE SUPPRESSION IN FREE SHEAR FLOWS UNDER ACOUSTIC EXCITATION
}

\author{
K.B.M.Q. Zaman and E.J. Rice \\ National Aeronautics and Space Administration \\ Lewis Research Center \\ Cleveland, Ohio 44135
}

\begin{abstract}
$\underline{\text { Abstract }}$
Acoustic excitation at certain high frequencies has been known to suppress large amplitude fluctuations otherwise occurring naturally in various free shear flows. The phenomenon has been observed in flows with initially laminar or transitional boundary layers. An experimental investigation is conducted to consider two possibilities in regards to the mechanism of the effect. (1) The natural shear layer is "self excited" by the instability waves already developed in the upstream boundary layer. This is overridden when the shear layer is excited at its maximally unstable mode, causing the observed decrease in the intensities downstream. (2) The upstream boundary layer is in a transitional or "buffeted laminar" state, characterized by large amplitude unsteady fluctuations, which force the large fluctuations downstream. Excitation "trips" the upstream boundary layer to full turbulence, reduces the unsteady fluctuations, and thus causes the observed suppression of the intensities throughout the flowfield. The present experimental results refute either of these possibilities to be the general mechanism of the effect.
\end{abstract}

\section{Introduction}

Artificial excitation can suppress large amplitude fluctuations otherwise occurring naturally in various free shear flows. ${ }^{1-4}$ The phenomenon has been observed in axisymmetric and plane jets as well as in plane mixing layers which are characterized by a nominally laminar or transitional state of the initial boundary layer near the point of separation. ${ }^{4}$ The suppression occurs globally over the entire cross section of the flow, moves the virtual origin of the flow downstream, and can be observed over a streamwise distance equal- ling several thousands of the initial momentum thickness $\left(\theta_{\mathrm{e}}\right)$. The excitation frequency $\left(f_{p}\right)$ producing the effect is "high" in comparison to the frequencies of unsteady fluctuations that characterize the regions of the flow where the effect manifests itself. Figure 1 is reproduced from Ref. 4 illustrating the effect in a circular and a plane jet. The velocity traces, presented with identical scales, demonstrate the remarkable suppression of the flow fluctuations under the excitation.

In Ref. 5, a similar effect of acoustic excitation was reported for a variety of wallbounded separated flows. In some of these flows, especially the ones involving transitory stall, the suppression of the unsteady fluctuations can be quite dramatic. Figure 2 shows this for a flow through a conical diffuser. ${ }^{5}$ The excitation reduces the intensity from more than 20 percent to less than 2 percent in the core of the flow. Figure 3 shows another example of the suppression effect for the flow over an airfoil. ${ }^{5}$ In this case, when the airfoil is held around the static stall angle, the flow undergoes an unsteady oscillation, characterized by the spectral peak around the unusually low nondimensional frequency of 0.02. Excitation at about $1 \mathrm{kHz}\left(\mathrm{f}_{\mathrm{p}} \mathrm{cSin} \alpha / \mathrm{U}_{\infty}=1.68\right)$ completely eliminates the unsteadiness and suppresses the flow fluctuations.

The suppression effect in the wallbounded separated flows appears morphologically similar to that in the free shear flows. The optimum excitation frequency in either case scales on the shear layer thickness near the point of separation. The effect in the former category of flows may not be due to a complete reattachment of the flow under the excitation. For example, in the airfoil case, the flow apparently remains fully separated under the excitation. Only the energetic 
coherent structures in the shear layer are eliminated or weakened which may even result in a loss in the lift coefficient. ${ }^{6}$ The effect in the wall-bounded separated flows, however, is obviously much more complex as separation and reattachment processes are involved. In any case, the potential for suppressing undesirable unsteadiness in a wall bounded separated flow, which is representative of the flows in many practical applications, provided the motivation for continuing to pursue the topic.

The objective of the present work is to make an effort to explain the mechanism of the suppression phenomenon. For this purpose, only the simpler case of a free shear layer is considered in the following.

\subsection{Previous Observations on the Mechanism of the Phenomenon}

In Ref. 4, it was observed that the excitation frequency producing the suppression phenomenon approximately corresponded to the maximally unstable disturbance frequency of the initial shear layer; the corresponding Strouhal number, $\mathbf{S t}_{\boldsymbol{\theta}}$, based on the initial shear layer momentum thickness $\left(\theta_{\mathrm{e}}\right)$ and the free stream velocity $\left(U_{e}\right)$, was about 0.017 . The effect, however, occurred over a range of the $\mathrm{St}_{\boldsymbol{\theta}}$ and a later investigation reported a somewhat higher optimum $\mathrm{St}_{\theta}$ when larger amplitudes of excitation were used. ${ }^{7}$ The latter work also reported a similar suppression effect observed computationally for a plane mixing layer.

In Ref. 4, it was observed furthermore that the "initial instability" frequency in the different shear layers was always substantially lower than the frequency component predicted to receive the maximum amplification rate. Spectral analysis showed that the natural disturbances growing the most in the initial region, and the subsequent roll up of the shear layer, occurred at $S t_{\theta} \approx 0.012$. In contrast, the predicted maximally unstable disturbance frequency corresponded to $\mathrm{St}_{\theta}=0.017,{ }^{8}$ which was confirmed experimentally by excitation at discrete frequencies and measuring the corresponding eigenfunctions. ${ }^{9}$ A lower Strouhal number for the "initial instability" was also reported in several other experiments (e.g., Ref. 10; see also Ref. 4).

The fact that the initial instability frequency is lower formed the basis for an explanation provided in Ref. 4 for the suppression effect. The naturally occurring lower frequency components persist farther downstream in the flow and attain larger amplitudes. That the lower frequency components should grow to a larger amplitude and persist farther downstream has been demonstrated, among others, in the experiment of Ref. 9. For example, an imposed disturbance at $\mathrm{St}_{\theta}=0.009$, in Fig. 16 of Ref. 9, can be seen to grow to a saturation amplitude that is about three times larger than that for a disturbance at $\mathrm{St}_{\theta}=0.017$. However, the streamwise distance where the saturation occurs for the former is about twice farther downstream than that for the latter. The fluctuation intensities in the natural shear layer, characterized by instability waves (or coherent structures) at the lower frequencies, are thus large. When an excitation at $\mathrm{St}_{\theta}=0.017$ is applied, the forced disturbance receives a fast amplification and saturation, resulting in a rapid roll up and earlier breakdown of the coherent structures. The experimental results show that this also inhibits the formation of the lower frequency energetic structures. The result is the suppression of the fluctuation intensities. It is noteworthy here that in the analysis of Ref. 11, higher Strouhal number instability waves, with shorter life-span, were shown to be inherently less efficient in the production of random turbulence. This should also contribute to the observed lower total intensity under the excitation.

It was conjectured in Ref. 4 that forcing the shear layer at its maximally unstable frequency inhibited the vortices from going through several stages of pairing, and this contributed further to the observed suppression effect. That vortex pairing is inhibited when the shear layer is excited near its 
maximally unstable mode was demonstrated by the experiment of Ho and Huang. ${ }^{12}$ It has been suggested, (in private communications with other researchers in the area), that this indeed could be the main reason for the observed suppression.

However, excitation at $\mathrm{St}_{\theta}=0.017$ does not totally eliminate vortex pairing. The data of Ref. 4, apparently for relatively larger amplitude forcing compared to that used in Ref. 12, showed that at least one stage of pairing took place. The number of stages of pairing within the length of the potential core of a circular jet may be expected to depend on the ratio of the jet diameter to the initial momentum thickness, $\mathrm{D} / \boldsymbol{\theta}_{\mathrm{e}^{\cdot}}{ }^{13}$ It becomes apparent from subsequent experimental results that multiple stages of pairing can take place under an excitation condition producing the suppression, and inhibition of vortex pairing may not be crucial to the phenomenon. ${ }^{14} \mathrm{~A}$ set of $u$ '-spectrum data from Ref. 14 is reproduced as Fig. 4. The data show suppression of the flow fluctuations in a circular jet when excited at $\mathrm{St}_{\theta}=0.017$; the total intensity at the measurement location reduced considerably as in Fig. 1. The spectra clearly show that the suppression is achieved in spite of the generation of three distinct subharmonics, indicating three stages of pairing, by the time the flow has reached the measurement location.

In Ref. 14, the suppression effect was compared with the effect of boundary layer tripping. It was observed that the excited shear layer was similar to the tripped flow. The initial condition effect, comparing tripped versus untripped flows, has been studied by many (e.g., Ref. 15). It has been known that for the initially "laminar" (untripped) case, the spread rate is faster, and the flow fluctuations in the developing regions are larger. The $\mathrm{St}_{\theta}=0.017$ excitation is found to suppress the fluctuations for the laminar case but at the most to levels that are typical of the turbulent case. It is as if the excitation trips the initial boundary layer and makes it turbulent.

\subsection{Deficiency in the Understanding}

A main question that has remained unanswered is why the "initial instability" appears to occur at a Strouhal number lower than that for the maximally unstable mode. Related to this, is a second question, remaining incompletely answered, as to why the flow fluctuations are higher in a shear layer with laminar or transitional initial condition. The second question can also be raised in connection with the studies on initial condition effect (tripped versus untripped). It appears that the latter question has not been addressed adequately in the related publications. A complete explanation of the suppression phenomenon is clearly linked to these questions.

\subsection{Proposed Mechanism}

In the present experimental investigation two hypotheses are considered.

(1) In the natural shear layer, the flow fluctuations are large due to a coupling between the Tollmien-Schlichting (T-S) waves in the upstream boundary layer and the free shear layer instability, i.e., the KelvinHelmholtz (K-H) waves, downstream. Excitation at the maximally unstable free shear layer instability frequency overrides this coupling and thus results in a suppression of the fluctuations.

Available data indicate that the flows in which the suppression effect is achieved involved upstream boundary layers that were apparently unstable; ${ }^{5}$ specifically, the momentum thickness Reynolds number, $\mathbf{R}_{\theta}$ for all the cited cases fell in the range of 200 to 700 . In this $R_{\theta}$ range, $T-S$ waves are likely to be developed, for a zero pressure gradient boundary layer. The $\mathrm{St}_{\theta}$ for the developed T-S waves, (upper branch of neutral stability curve, Ref. 16), should correspond to a $\mathrm{St}_{\theta}$ range of 0.007 to 0.009 . The developed T-S waves would excite the $\mathrm{K}-\mathrm{H}$ waves. The flow downstream is thus not only in a state of "self excitation" but also the excited waves are at a lower Strouhal number. As discussed in 
Section 1.1, this would thus explain the large fluctuations observed in the flow. While the T-S waves occur in the $\mathrm{St}_{\theta}$ range of 0.007 to 0.009 in the upstream boundary layer, an increase in the momentum thickness, by the distance the boundary layer profile relaxes to a free shear layer profile, ${ }^{9,10}$ might also explain the $\mathrm{St}_{\theta} \approx 0.012$ value for the observed free shear layer initial instability.

When the flow is forced at $\mathrm{St}_{\theta} \approx 0.017$, all other disturbances are precluded. The forced disturbance receives a rapid growth by the free shear layer. However, it "saturates" earlier in $x,{ }^{9}$ and consequently, the suppression effect is achieved farther downstream. The fact that a forced disturbance precludes other disturbances, which were occurring naturally, is a result noted in various experiments. ${ }^{4,13}$ However, the fluid dynamical basis for this remains unclear, and thus, represents a weak link in the above reasoning.

(2) The second possibility is based on the idea, as alluded to before, that the boundary layer prior to the point of separation is "tripped" to full turbulence under the excitation. In the natural flow under consideration, the initial boundary layer is transitional or in a "buffeted laminar" state and therefore involves fluctuation intensities larger than that encountered in a fully turbulent boundary layer. ${ }^{17,18}$ The large initial fluctuations drive the unsteadiness in the flow causing the larger intensities downstream. When acoustically forced at the high frequency, the boundary layer is excited and becomes turbulent.
Consequently, the initial fluctuation level reduces resulting in a commensurate reduction in the intensities downstream.

An experiment was conducted to assess the validity of these two possibilities. These results are described in the following.

\section{Experimental Procedure}

The experiments were conducted in an axisymmetric jet facility, schematically shown in Fig. 5. The flow passed through a 76-cm diameter plenum chamber and then through two stages of contraction before exiting through a 5.08-cm diameter nozzle. The nozzle had a $1.27 \mathrm{~cm}$ long cylindrical section prior to the exit. A $15.2 \mathrm{~cm}$ or a $30.4 \mathrm{~cm}$ long cylindrical extension, with option for boundary layer trip (see Fig. 5), could be added to the nozzle to obtain thicker afflux boundary layers. Measurements were done for four cases (see Table $I$ ). Where $M_{e}$ is the jet Mach number at the nozzle exit, $\theta_{\mathrm{e}}$ the momentum thickness, $\mathrm{R}_{\theta}$ the Reynolds number based on $\theta_{e}, \mathrm{H}_{12}$ the shape factor, and $u_{\max }$, the maximum fluctuation intensity in the boundary layer. Since Case 1 involves a low enough $R_{\theta}$, the upstream boundary layer should be stable. In this case there should not be any T-S waves to drive the $K-H$ waves and, therefore, the suppression phenomenon should not be observed if hypothesis (1) were true. If hypothesis (2) were correct, at least in Case 3 the boundary layer near the jet exit would be expected to become fully turbulent under the excitation.

TABLE I.--MEASUREMENT CONDITIONS

\begin{tabular}{|c|c|r|r|l|r|l|}
\hline Case & $\mathrm{M}_{\mathrm{e}}$ & $\begin{array}{c}\theta_{\mathrm{e}}, \\
\text { in. }\end{array}$ & $\mathrm{R}_{\theta}$ & $\mathrm{H}_{12}$ & $\mathrm{u}_{\max } / \mathrm{U}_{\mathrm{e}}$ & \multicolumn{1}{|c|}{ b.l. state } \\
\hline 1 & 0.02 & 0.0086 & 100 & 2.3 & 0.02 & laminar \\
2 & .054 & .0111 & 348 & 2.2 & .03 & laminar \\
3 & .1 & .0153 & 920 & 2.0 & .14 & intermittent \\
4 & .1 & .0267 & 1565 & 1.49 & .10 & turbulent \\
\hline
\end{tabular}


TABLE II.-EXCITATION AMPLITUDE AND PROBE LOCATION

\begin{tabular}{|c|c|c|c|c|}
\hline Case & \multicolumn{1}{|c|}{$\mathrm{R}_{\theta}$} & $\mathrm{x} / \theta_{\mathrm{e}}$ & $(\mathrm{D} / 2-\mathrm{y}) / \theta_{\mathrm{e}}$ & $\begin{array}{c}\mathrm{u}_{\mathrm{fe}}^{\prime} / \mathrm{U}_{\mathrm{e}} \\
\text { percent }\end{array}$ \\
\hline 1 & 100 & 400 & 30 & 0.3 \\
2 & 348 & 400 & 35 & .3 \\
3 & 920 & 390 & 32 & .2 \\
4 & 1565 & 225 & 20 & .2 \\
\hline
\end{tabular}

\section{Results}

The mean velocity (U) and fluctuation intensity ( $\left.u^{\prime}\right)$ profiles for the exit boundary layer are shown in Fig. 6. Note that the intensity for the $R_{\theta}=920$ case is much higher than that in the fully turbulent, $\mathrm{R}_{\theta}=\mathbf{1 5 6 5}$ case. The u'-spectra measured at the 60 percent velocity point in the boundary layer are shown in Fig. 7(a) for the two higher $R_{\theta}$ cases. For $R_{\theta}=920$, the spectrum is characterized by energy at low frequencies. The intermittent switching of the velocity profile from laminar to turbulent states yields the large amplitude fluctuations when measured at a fixed point within the boundary layer.

The corresponding velocity spectra for the two lower $R_{\theta}$ cases are shown in Fig. 7(b). These measurements needed special attention to avoid electronic noise and the signals directly from the anemometers were analyzed without a linearizer. The amplitudes are shown in rms millivolts. The spikes at the harmonics of line noise at $60 \mathrm{~Hz}$ are still quite prominent which is a typical problem in low level turbulence measurements. In Fig. 7(b) one finds that even at the lowest $R_{\theta}$, there is energy at low frequencies. This is likely to be due to boundary layer separation somewhere upstream in the flow facility. Away from the boundary layer, in the core of the jet, however, the low frequency components disappeared. The turbulence intensity, in the core of the jet at the nozzle exit, for all four cases, was estimated to be less than 0.15 percent.

Figure 8 shows the excitation effect on the fluctuation intensity measured at a fixed location downstream. The ordinate is the ratio of the intensities with and without the excitation. The horizontal line for each case represents the value of unity. Values less than unity indicate suppression of the fluctuations under the excitation. For each data point in each curve the excitation amplitude at the exit plane of the jet, $\left(u_{f e}^{\prime}\right.$, the rms fundamental at the excitation frequency), was held approximately constant. The amplitudes and the probe locations for the four cases are listed in Table II. The results are similar to that reported in Ref. 4. For the initially turbulent case, very little suppression is achieved, as also observed in Ref. 4. Of significance is the fact that suppression is achieved even at $\mathrm{R}_{\theta}=100$. This seems to disprove hypothesis (1).

In Fig. 9 radial profiles of the fluctuation intensities are compared with and without excitation for $R_{\theta}=100,348$, and 920 cases, at the corresponding $\mathrm{x}$-locations as listed in Table II. These data demonstrate that the suppression, even though not as much as in Fig. 2 or the cases reported in Ref. 4, is a global effect and is not characteristic of a particular probe location. In Fig. 10(a), evolution of the $u$ '-spectra with downstream distance is shown for the $\mathrm{R}_{\theta}=348$ case. In a jet facility, there are unavoidable background disturbances and these are amplified variably by the shear layer according to its stability characteristics. The spectral evolution here is quite similar to that reported by Cohen and Wygnanski. ${ }^{19}$ It can be observed from these data that the disturbance at $\mathrm{St}_{\theta} \approx 0.013$ is amplified the most by the shear layer. The subsequent roll up of the shear layer may be expected at this frequency. Note that the 
spectrum just downstream of the nozzle exit does not contain any large amplitude spike at $\mathrm{St}_{\theta} \approx 0.013$. This clearly indicates that the shear layer is not being excited by disturbances already developed in the boundary layer.

Evidence of the T-S wave growth in the upstream boundary layer was further looked for. In order to do this the low frequency energy from the spectra was filtered out and the signal was amplified. The u'-spectra measured at four $x$-locations are shown in Fig. 10(b). Unfortunately, the amplifiers also amplified the electronic noise possibly with an additional noise contribution from themselves. However, a close inspection of the data for $x / \theta_{e}=-55$ and $-2 \overline{7}$ should convince one that significant amplification of any spectral component has not taken place in the boundary layer. The amplification essentially starts downstream of the nozzle lip, in the shear layer. These results are evidence that hypothesis (1) may not be correct.

Figure 11 shows the $U$ - and $u$ '-profiles in the exit boundary layer, with and without excitation, for the $R_{\theta}=920$ case. The excitation frequency and amplitude are the same as used in Fig. 9 which also represent the corresponding optimum excitation condition in Fig. 8. Clearly, the U- and u'-profiles at the nozzle exit are essentially unaffected by the excitation. The corresponding u'-spectra with and without excitation in the exit boundary layer are shown in Fig. 12. Except for the spike at the excitation frequency, the two spectra are essentially identical. These data prove that the boundary layer at the nozzle exit has not been turned turbulent by the excitation. Thus, hypothesis (2) is also not valid as a general rule.

\section{Concluding Remarks}

The upstream boundary layer is not characterized by developed T-S waves in the cases considered. Even though the nozzle has a cylindrical section prior to the exit, a small favorable pressure gradient may exist which most likely renders the boundary layer stable in the $R_{\theta}$ range under consideration.

"Acoustic tripping" of the upstream boundary layer cannot explain the suppression phenomenon under consideration. Clearly the upstream boundary layer is affected very little and the imposed disturbance is amplified almost exclusively by the separated shear layer. Further effort to explain the "turbulence suppression" phenomenon must focus on the separated shear layer.

\section{$\underline{\text { References }}$}

1. Vlasov, Y.V. and Ginevskiy, A.S., "Generation and Suppression Turbulence in an Axisymmetric Turbulent Jet in the Presence of an Acoustic Influence," NASA TT-F-15, 721, 1974.

2. Petersen, R.A., Kaplan, R.E., and Laufer, J., "Ordered Structures and Jet Noise," NASA CR-134733, 1974.

3. Rockwell, D.O., "External Excitation of Planar Jets," Journal of Applied Mechanics, Vol. 39, 1972, pp. 883-890.

4. Zaman, K.B.M.Q. and Hussain, A.K.M.F., "Turbulence Suppression in Free Shear Flows by Controlled Excitation," Journal of Fluid Mechanics, Vol. 103, 1981, pp. 133-159.

5. Zaman, K.B.M.Q., "A steadying effect of acoustic excitation on transitory stall," AIAA Paper 91-0043, Jan. 1991 (also, NASA TM-103689).

6. Zaman, K.B.M.Q., Bar-Sever, A., and Mangalam, S.M., "Effect of acoustic excitation on the flow over a low- $R_{e}$ airfoil," Journal of Fluid Mechanics, Vol. 182, 1987, pp. 127-148. 
7. Nallasamy, M. and Hussain, A.K.M.F., "Numerical study of the phenomenon of turbulence suppression in a plane shear layer," Proceedings, 4th Symposium on Turbulent Shear Flows, Pennsylvania State University, University Park, PA, 1984, pp. 169-181.

8. Michalke, A., 1965, "On spatially growing disturbances in an inviscid shear layer," Journal of Fluid Mechanics, Vol. 23, 1965, pp. 521-544.

9. Freymuth, P., "On transition in a separated laminar boundary layer," Journal of Fluid Mechanics, Vol. 25, 1966, pp. 683-704.

10. Drubka, R.E., "Instabilities in near field of turbulent jets and their dependence on initial conditions and Reynolds number," Ph.D. Thesis, Illinois Institute of Technology, 1981.

11. Mankbadi, R.A., "On the interaction between fundamental and subharmonic instability waves in a turbulent round jet," Journal of Fluid Mechanics, Vol. 160, 1985, pp. 385-419.

12. Ho, C.-M. and Huang, L.-S., "Subharmonics and vortex merging in mixing layers," Journal of Fluid Mechanics, Vol. 119, 1982, pp. 443-473.

13. Kibens, V., "Discrete Noise Spectrum Generated by an Acoustically Excited Jet," AIAA Paper 79-0592, Jan. 1979.
14. Zaman, K.B.M.Q., "Far-field noise of a subsonic jet under controlled excitation," Journal of Fluid Mechanics, Vol. 152, 1985, pp. 83-111.

15. Batt, R.G., "Some measurements on the effect of tripping the two-dimensional shear layer," AIAA Journal, Vol. 13, 1975, pp. 245-247.

16. Saric, W.S. and Nayfeh, A.H., "Nonparallel stability of boundary-layer flows," Physics of Fluids, Vol. 18, 1975, pp. 945-950.

17. Purtell, L.P., Klebanoff, P.S., and Buckley, F.T., "Turbulent Boundary Layer at Low Reynolds Number," Physics of Fluids, Vol. 24, 1981, pp. 802-811.

18. Sohn, K.H., O'Brien, J.E., and Reshotko, E., "Some characteristics of bypass transition in a heated boundary layer," Proceedings, 7th Symposium on Turb. Shear Flows, Vol. 1, Pennsylvania State University, University Park, PA, 1989, pp. 2.4.4-2.4.6 (also, NASA TM-102126).

19. Cohen, J. and Wygnanski, I., "The evolution of instabilities in the axisymmetric jet. Part 1. The linear growth of disturbances near the nozzle," Journal of Fluid Mechanics, Vol. 176, 1987, pp. 191-219. 
Circular jet
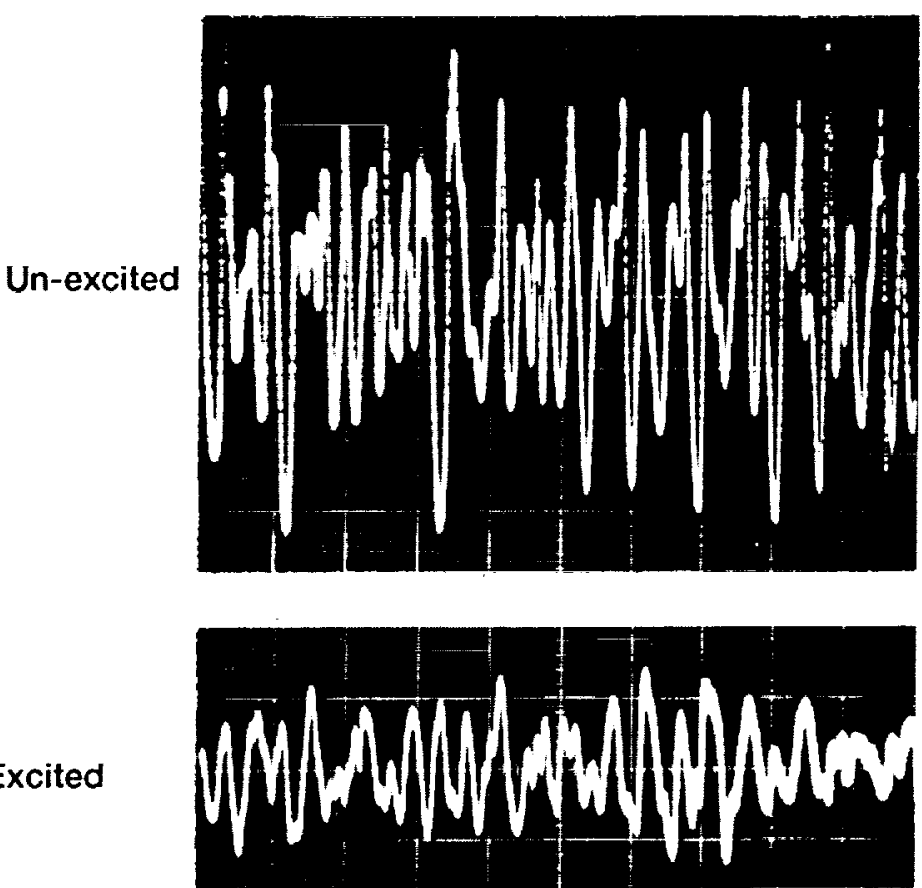

Plane jet
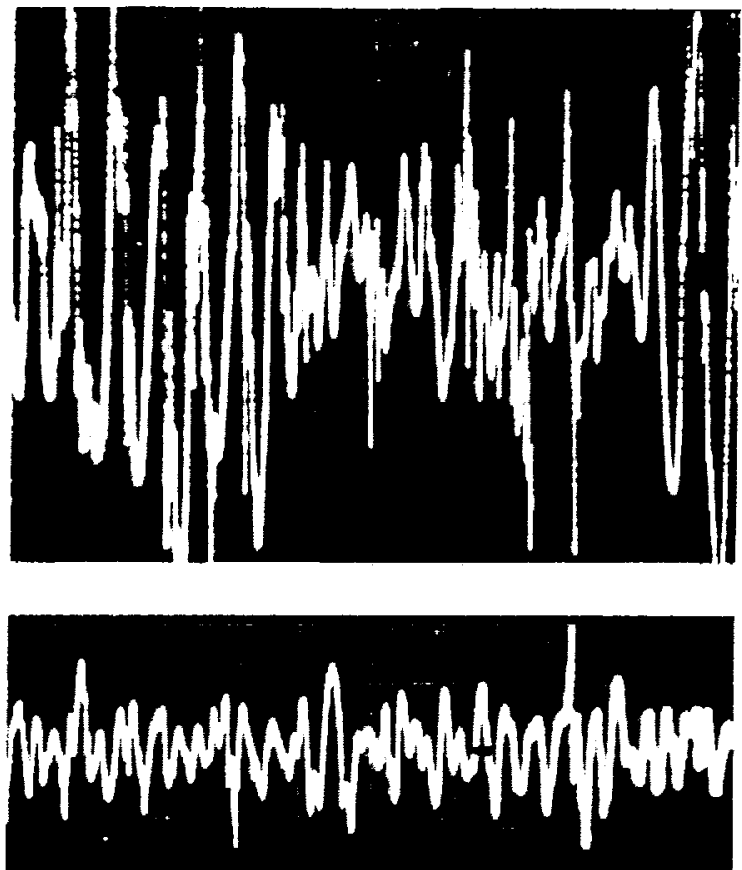

Figure 1.-Oscilloscope traces of hot-wire $u(t)$ signals on the jet axis at $x=10 \mathrm{~cm}$, from Ref. 4 . For the circular jet, $D=2.54 \mathrm{~cm}, U_{e}=12.7 \mathrm{~ms}-1, f_{p}=1050 \mathrm{~Hz}$; for the plane jet, $w=3.18 \mathrm{~cm}, U_{e}=22 \mathrm{~ms}^{-1}$, $f_{p}=1780 \mathrm{~Hz}$. Each trace covers $100 \mathrm{~ms}$.
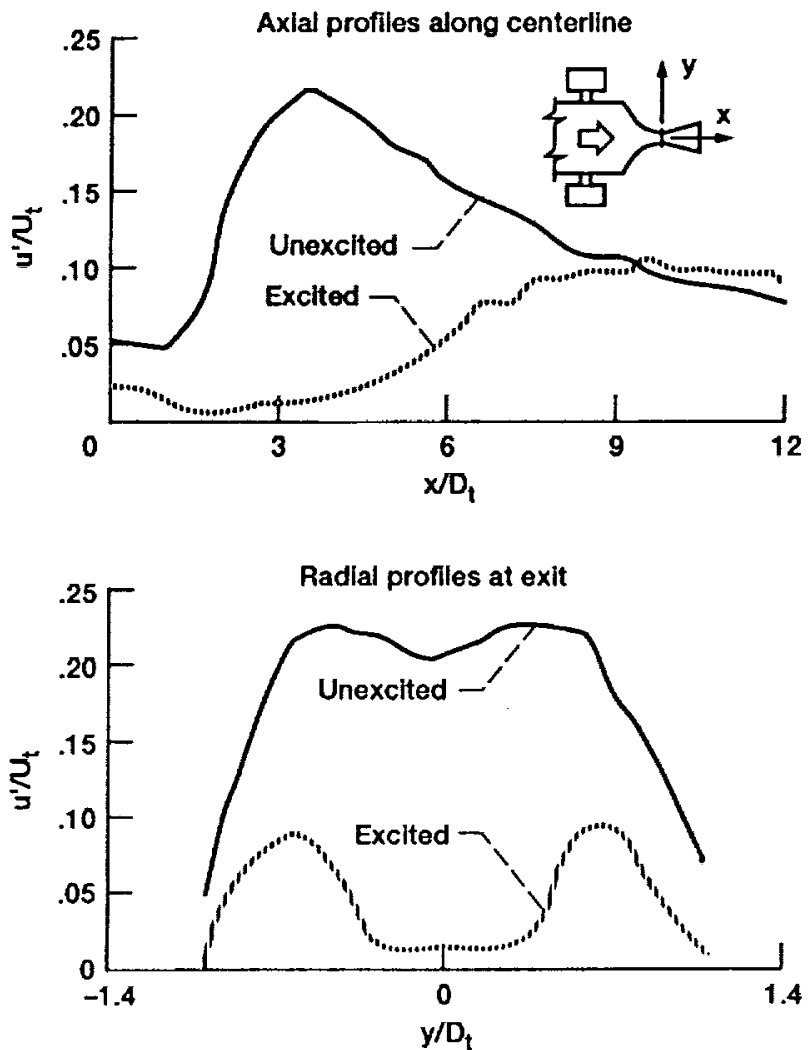

Figure 2.-Profiles of total r.m.s. fluctuation intensity, with and without excitation for the flow through a $20^{\circ}$ diffuser, from Ref. 5. $M_{t}=0.075, f_{p}=1.1 \mathrm{kHz}$; subscript $t$ denotes conditions at throat $(x=0)$.

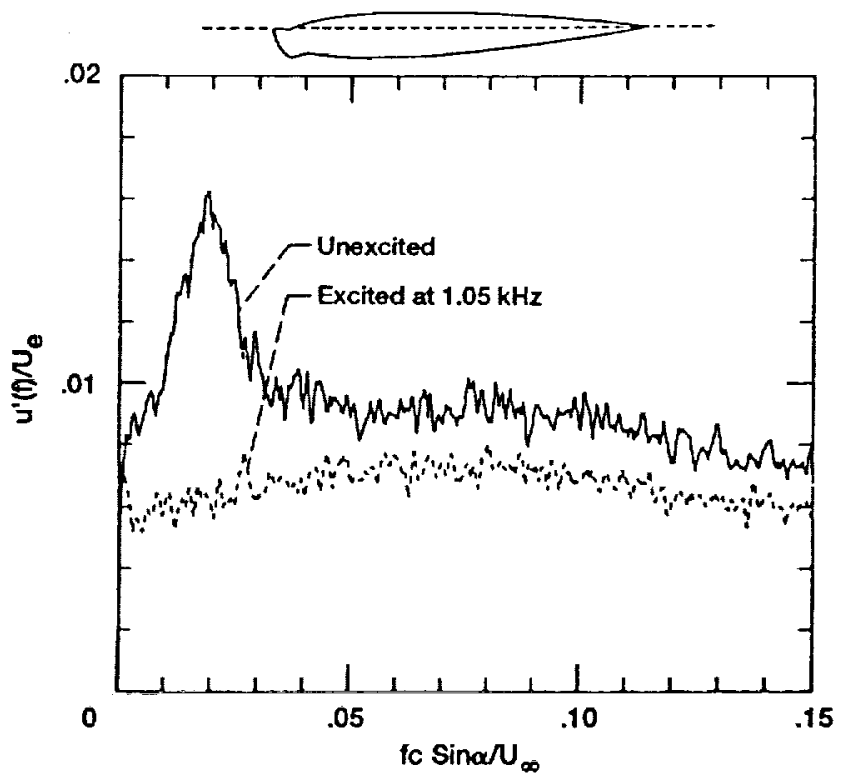

Figure 3.-Wake velocity spectra with and without excitation, from Ref. 5. "iced" airfoil at angle of attack, $\alpha=8.5^{\circ}$, and chord Reynolds number, $R_{c}=10^{5}$. 


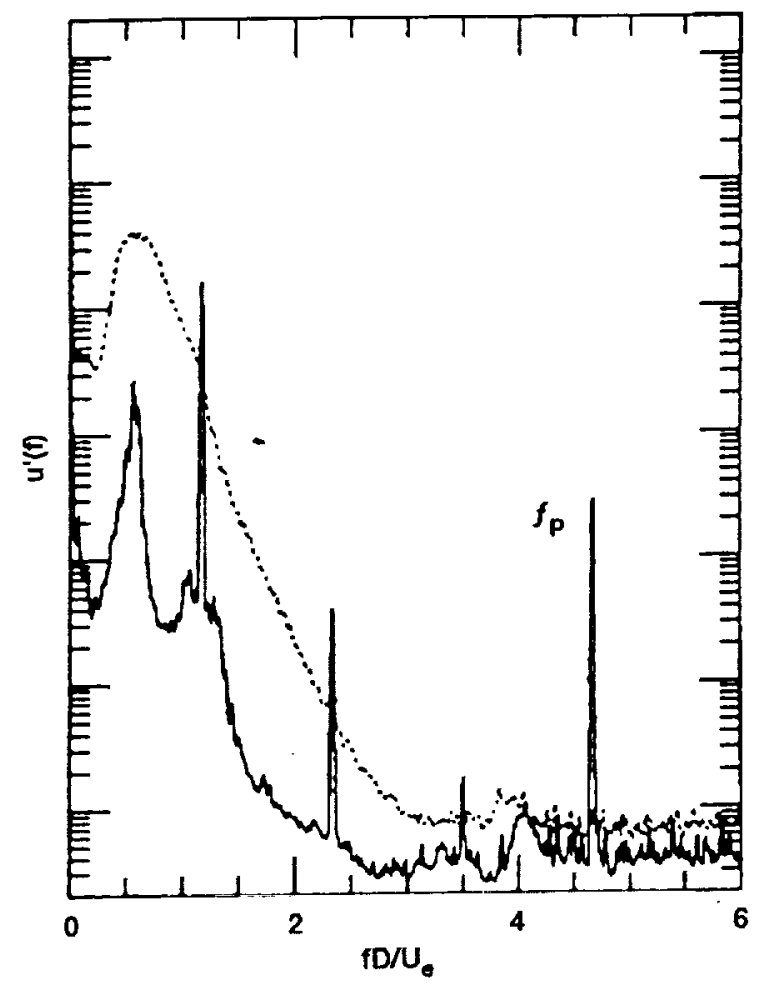

Figure 4.- $u^{\prime}$-spectrum showing suppression in a $2.54 \mathrm{~cm}$ jet, from Ref. 14. Dashed curve for unexcited case, solid curve for excited case; $S t_{\theta}=0.017, u^{\prime}{ }_{t e} / U_{\theta}=$ $0.03 \%, M_{e}=0.12$; hot-wire at $X / D=1.5$ on jet axis.

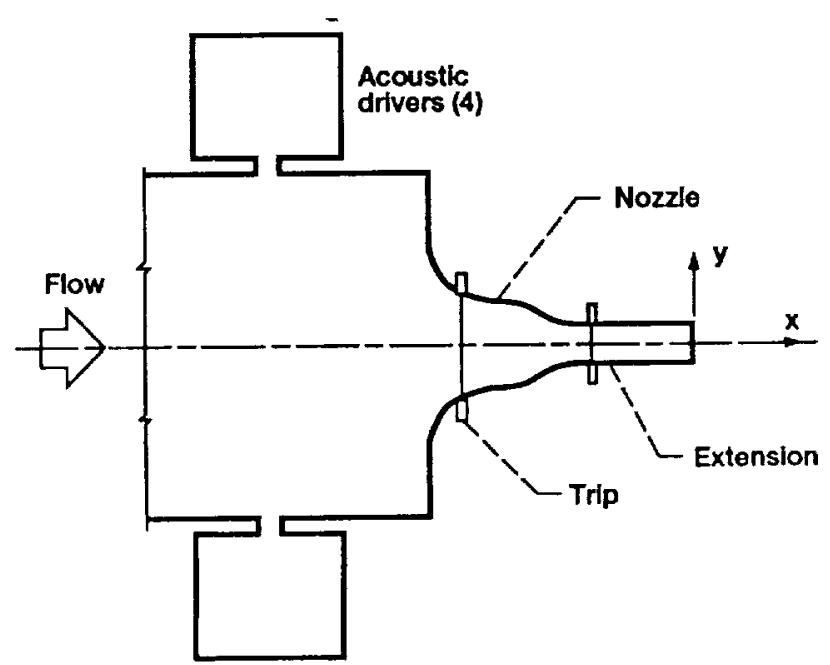

Figure 5.-Schematic of flow facility.
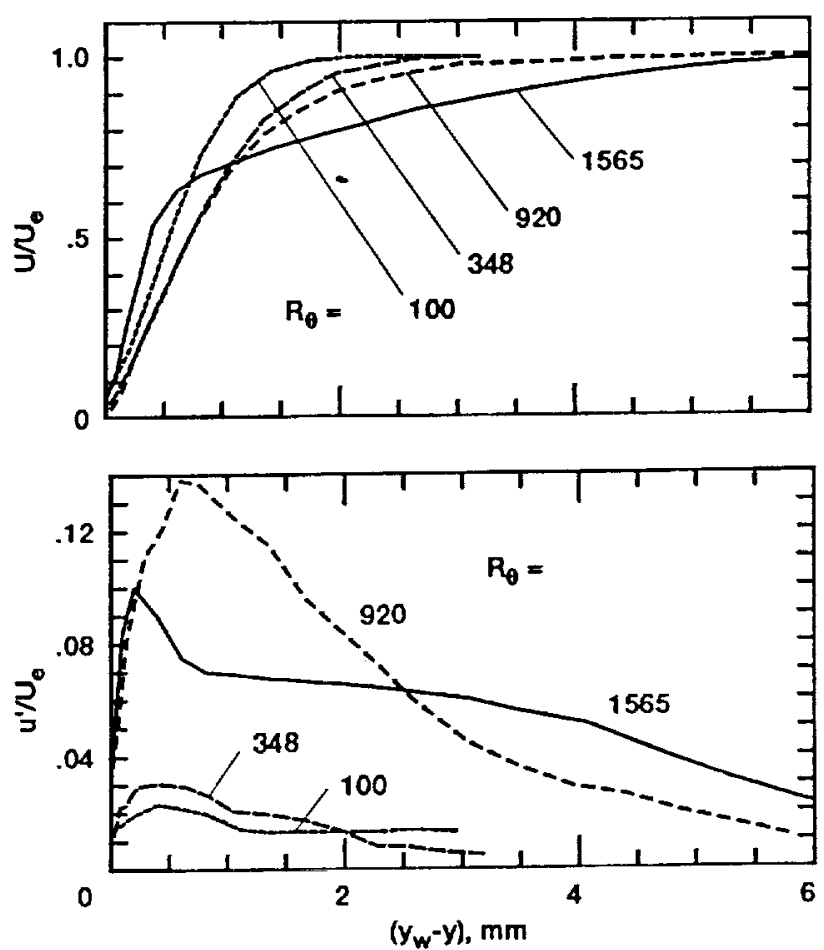

Figure 6.-U- and $u^{\prime}$-profiles in the exit boundary layer, measured at about $1 \mathrm{~mm}$ downstream of nozzle lip, for Indicated $R_{\theta}$ 

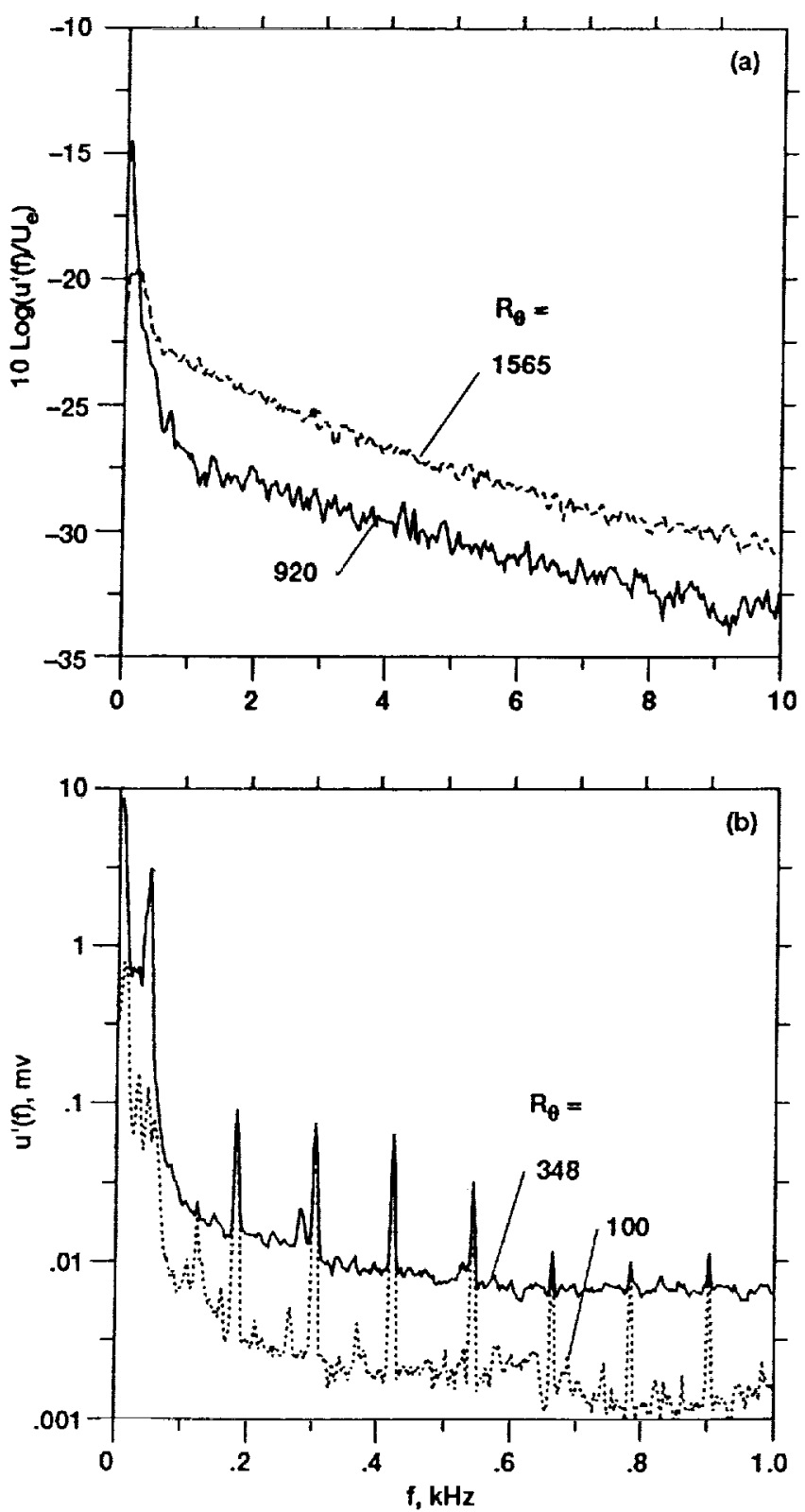

Figure 7.- $\mathrm{u}^{\prime}$-spectra in the exit boundary layer at 60 percent velocity point for indicated $R_{\theta}$.

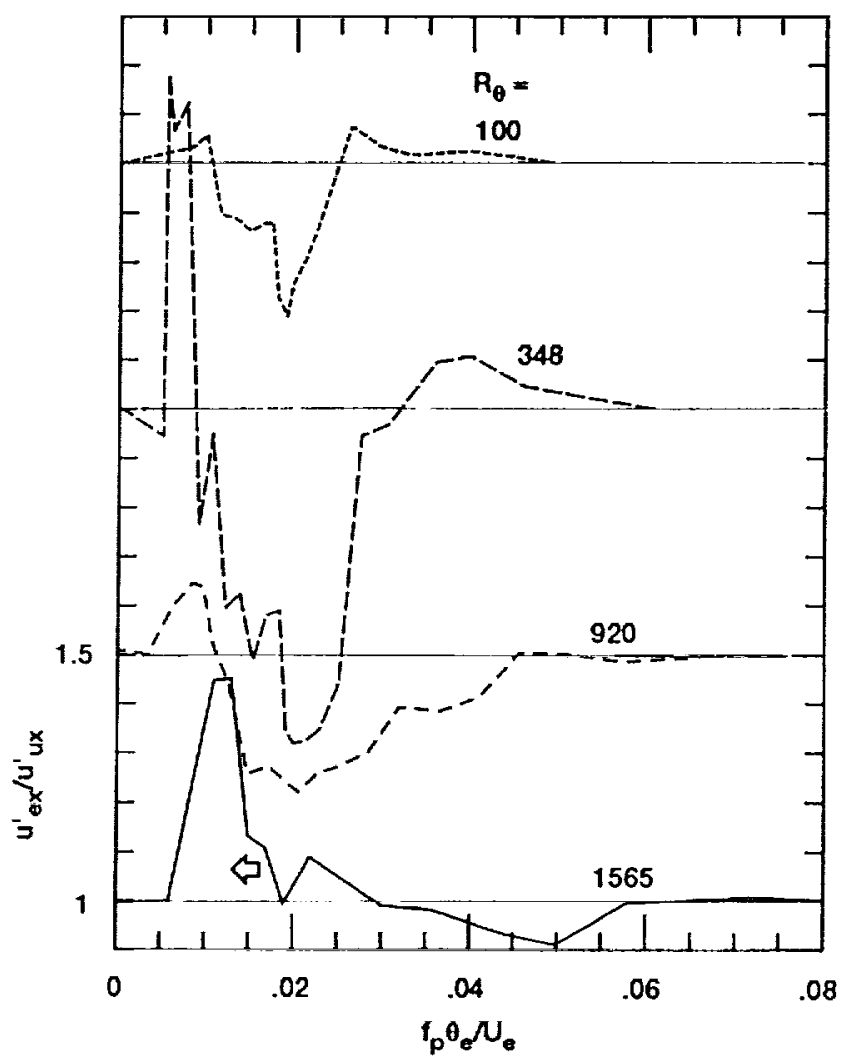

Figure 8.-Ratlos of fluctuation intensities with and without excitation measured at a fixed downstream location, for the four cases. Successive curves are staggered by one major ordinate division.

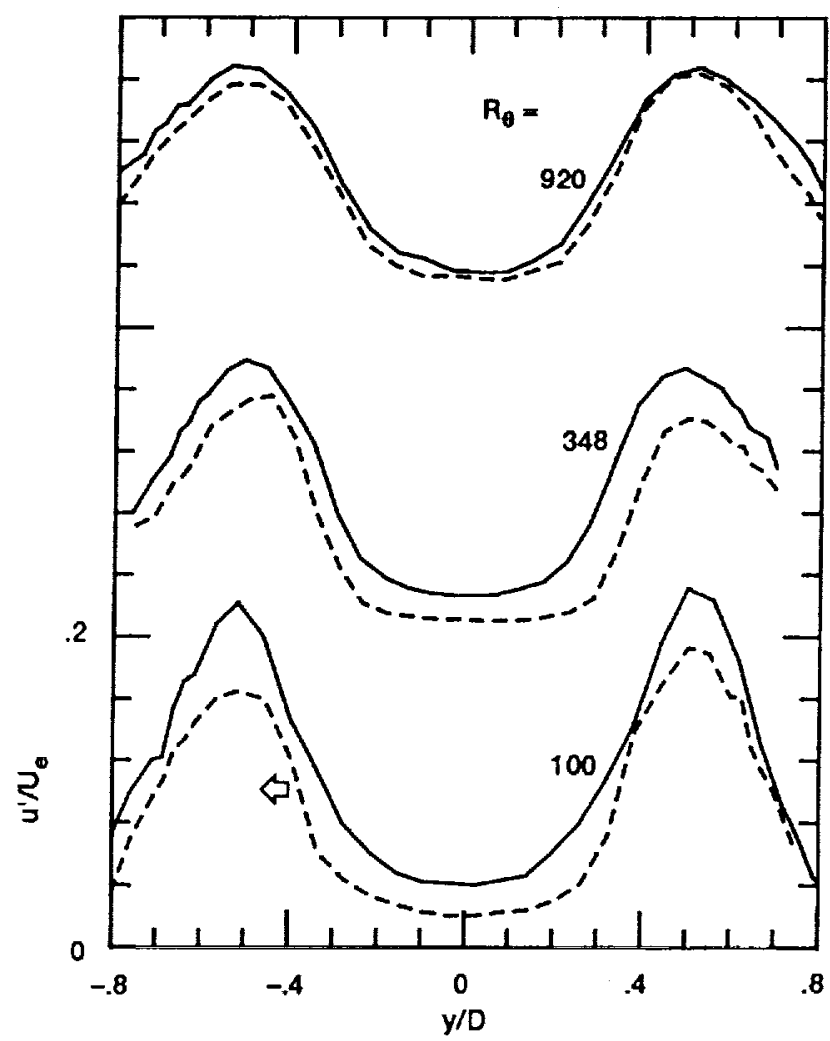

Figure 9.-Diametral profiles of $u^{\prime}$ for indicated $R_{t}$. Pairs of curves staggered by one major division. Solid line, unexcited, dashed line, excited. 

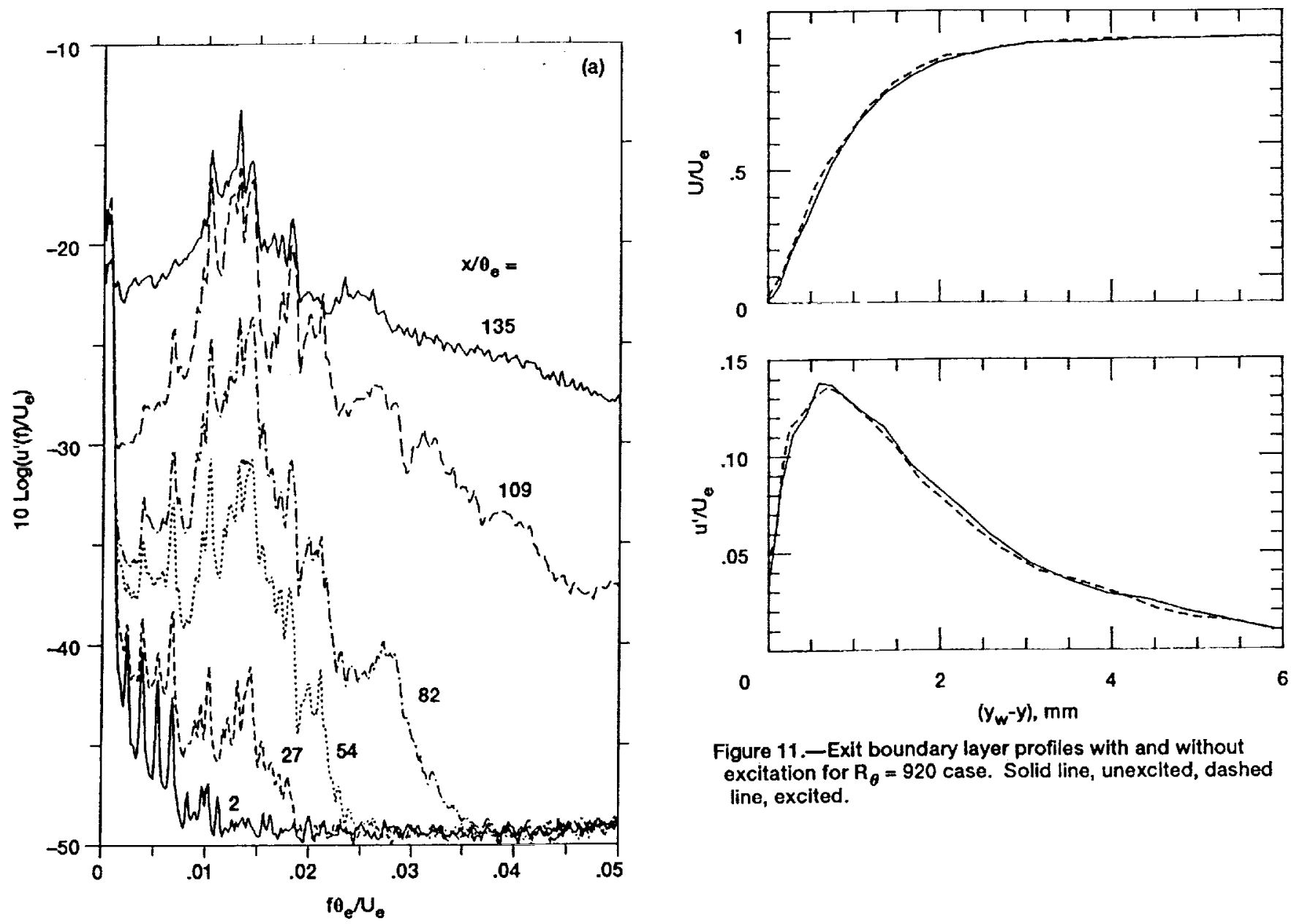

Figure 11.-Exit boundary layer profiles with and without excitation for $R_{\theta}=920$ case. Solid line, unexclted, dashed line, excited.

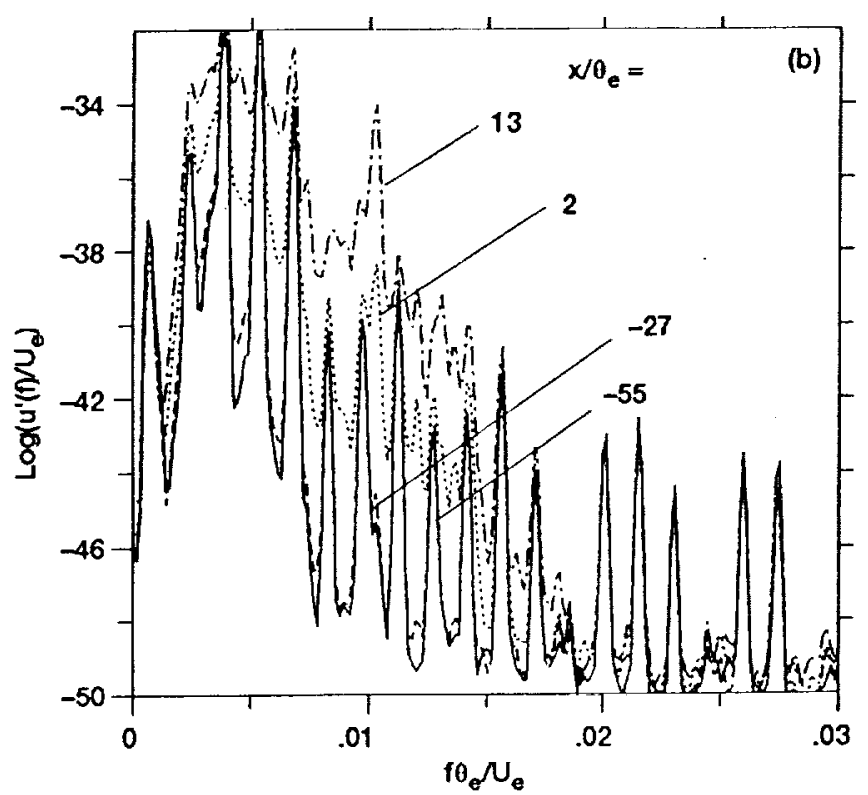

Figure 10.-u'-spectra at 60 percent velocity point at different $x / 0_{e}$, for the $R_{\theta}=348$ case. (a) Data downstream from exit. (b) Data around and upstream of exit with arbitrary vertical scale; signals high pass filtered with $200 \mathrm{~Hz}$ cutoff.

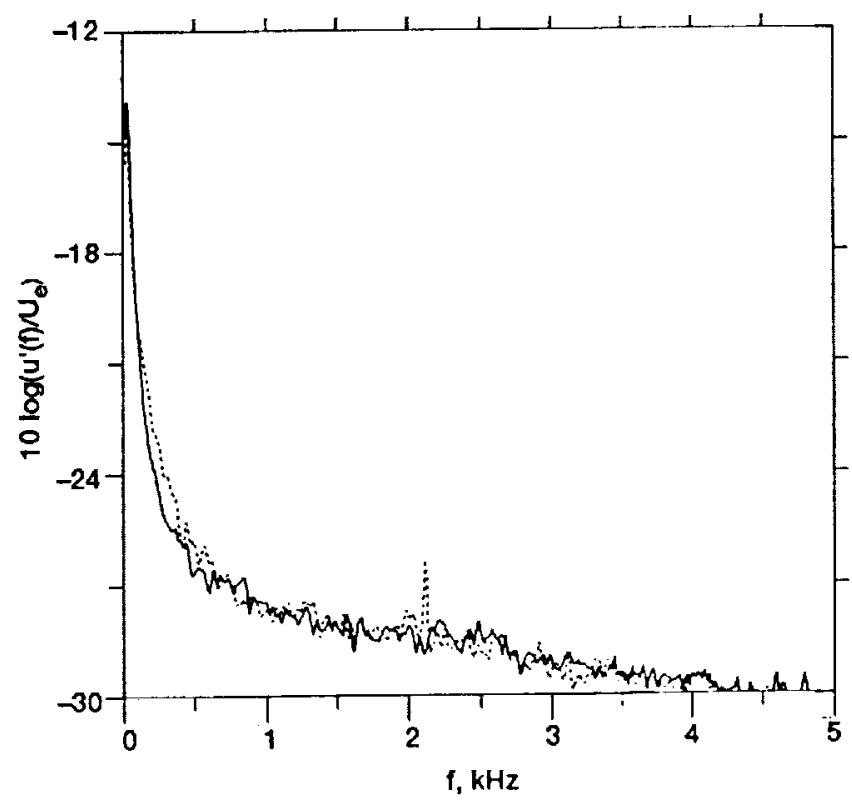

Figure 12.- $u$-spectra at 60 percent velocity point in the exit boundary layer for the $R_{\theta}=920$ case. Solid line, unexcited, dashed line, excited. 


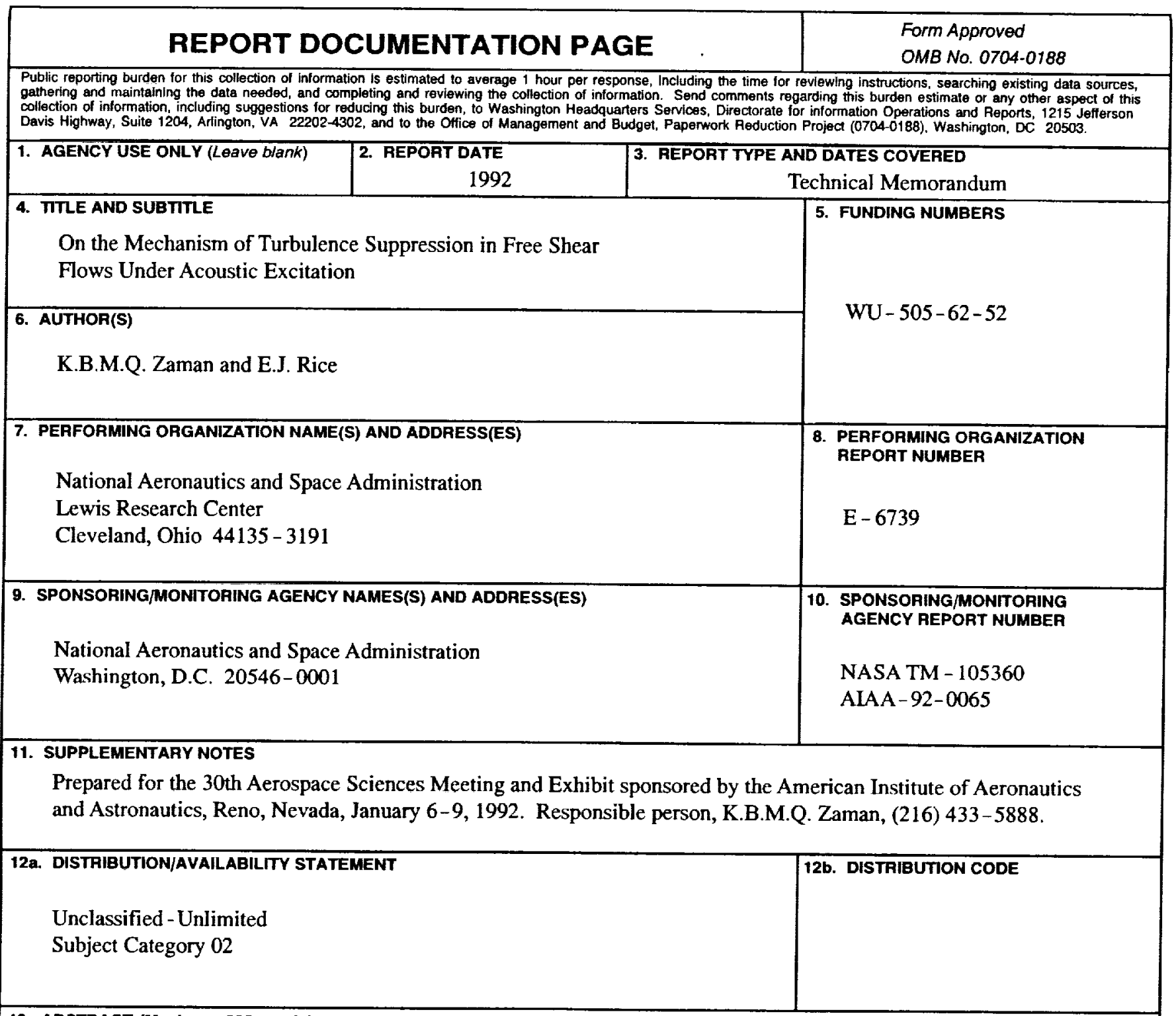

\section{ABSTAACT (Maximum 200 words)}

Acoustic excitation at certain high frequencies has been known to suppress large amplitude fluctuations otherwise occurring naturally in various free shear flows. The phenomenon has been observed in flows with initially laminar or transitional boundary layers. An experimental investigation is conducted to consider two possibilities in regards to the mechanism of the effect. (1) The natural shear layer is "self excited" by the instability waves already developed in the upstream boundary layer. This is overridden when the shear layer is excited at its maximally unstable mode, causing the observed decrease in the intensities downstream. (2) The upstream boundary layer is in a transitional or "buffeted laminar" state, characterized by large amplitude unsteady fluctuations, which force the large fluctuations downstream. Excitation "trips" the upstream boundary layer to full turbulence, reduces the unsteady fluctuations, and thus causes the observed suppression of the intensities throughout the flowfield. The present experimental results refute either of these possibilities to be the general mechanism of the effect.

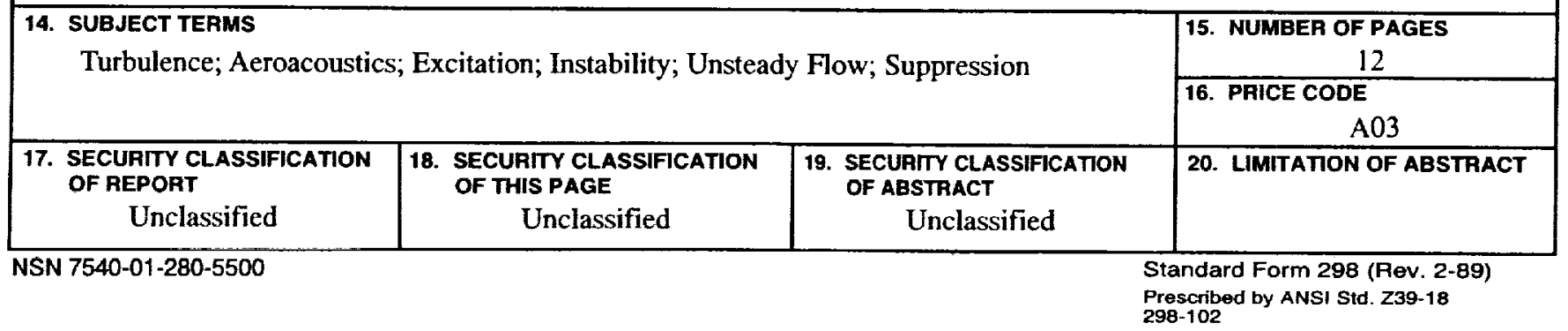

